\title{
Discovery of lung surface intersegmental landmarks by three-dimensional reconstruction and morphological measurement
}

\author{
Yuyang Xu ${ }^{1,2,3 \#}$, Fanyi Gan ${ }^{1,3 \#}$, Chunchao Xia ${ }^{4}$, Zihuai Wang ${ }^{1}$, Kejia Zhao ${ }^{1}$, Chuan Li $^{1}$, Jiandong Mei ${ }^{1,3}$, \\ Chengwu Liu ${ }^{1,3}$, Hu Liao ${ }^{1,3}$, Qiang Pu ${ }^{1,3}$, Lunxu Liu ${ }^{1,2,3}$ \\ ${ }^{1}$ Department of Thoracic Surgery, West China Hospital, Sichuan University, Chengdu 610041, China; ${ }^{2}$ West China Precision Medicine Industrial \\ Technology Institute, Chengdu 610041, China; ${ }^{3}$ Western China Collaborative Innovation Center for Early Diagnosis and Multidisciplinary Therapy \\ of Lung Cancer, Chengdu 610041, China; ${ }^{4}$ Department of Radiology, West China Hospital, Sichuan University, Chengdu 610041, China \\ Contributions: (I) Conception and design: L Liu; (II) Administrative support: Y Xu, F Gan; (III) Provision of study materials or patients: Y Xu, F Gan, \\ C Xia; (IV) Collection and assembly of data: F Gan, Y Xu, K Zhao; (V) Data analysis and interpretation: Y Xu, F Gan, Z Wang; (VI) Manuscript \\ writing: All authors; (VII) Final approval of manuscript: All authors. \\ \#These authors contributed equally to this work. \\ Correspondence to: Lunxu Liu, MD, PhD, FRCS. Department of Thoracic Surgery, West China Hospital, Sichuan University, No. 37 Guoxue Alley, \\ Chengdu 610041, China. Email: lunxu_liu@aliyun.com.
}

Background: The lack of anatomic landmarks between segments on the lung surface makes the identification of intersegmental planes one of the greatest challenges in anatomic segmentectomy. Therefore, with the aim to determine the landmarks of intersegmental planes on the lung surface, we used threedimensional (3D) reconstruction and morphological measurement techniques to reconstruct stereoscopic models of all pulmonary segments, and measured the length of each segment on the lung surface along the lobe's anatomic landmark lines.

Methods: We downloaded the primary computed tomography (CT) scan data of 619 patients and imported them into a $3 \mathrm{D}$ reconstruction system, which could automatically reconstruct the $3 \mathrm{D}$ model of the tracheabronchi system. We manually reconstructed the intersegmental veins to ensure the accuracy of segmental boundary. The 3D models of pulmonary segments could be reconstructed based on the bronchial tree and the pathways of the intersegmental veins. We then measured the length of each segment on the lung surface along the lobe's anatomic landmark lines and calculated the proportions between these lengths.

Results: Complete 3D segmental models were successfully reconstructed in 500 patients (241 male and 259 female), and the lengths of every segment on the lung surface along the lobe's anatomic landmark lines were measured. Our data revealed that the length of each segment on lung surface varied among individuals. However, the proportions between these lengths stayed constant, even when stratified by gender, age, height, and weight.

Conclusions: We discovered that the proportion between the lengths of adjacent segments on the lung surface stayed constant. The constant proportion reflected and uncovered the lung surface intersegmental landmarks, which could help direct surgeons to identify intersegmental planes during anatomic segmentectomy in an easy and safe way without additional cost.

Keywords: Segmentectomy; 3D reconstruction; morphological measurement; intersegmental landmarks; intersegmental plane identification

Submitted Nov 19, 2019. Accepted for publication Dec 09, 2019.

doi: $10.21037 /$ tlcr.2019.12.21

View this article at: http://dx.doi.org/10.21037/tlcr.2019.12.21 


\section{Introduction}

Since the development and widespread application of computed tomography (CT) imaging, the early detection of small-sized lung cancers that are more likely to have inert biological has dramatically increased of. Meanwhile recent studies have provided some evidence supporting anatomic segmentectomy as a procedure on par with lobectomy for the treatment of small-sized lung cancers (1-5).

The concept of "broncho-pulmonary segment" was first introduced in 1932 (6). However, over the past decades, no population-based studies have illuminated the spatial relationship among the segments or investigated the marks of intersegmental planes on the lung surface. The absence of anatomic landmarks between segments makes the identification of intersegmental planes one of the most challenging impediments in anatomic segmentectomy, and methods that can easily identify the intersegmental planes remain elusive.

We therefore attempted to use the three-dimensional (3D) reconstruction technique, which has been proven to be valuable in the preoperative assessment of anatomic segmentectomy $(7,8)$, to reconstruct stereoscopic images of pulmonary segments. In combination with the morphological measurement technique, we attempted to investigate the length of segments on the lung surface along lobe boundary lines and determine their relationships with the aim of identifying effective lung surface intersegmental landmarks.

\section{Methods}

\section{Patient population}

From January to December, 2017, patients with pulmonary nodules less than $2 \mathrm{~cm}$, who had undergone thoracic highresolution computed tomography (HRCT) with a slice thickness of $1 \mathrm{~mm}$ and pulmonary angiography in our hospital, were enrolled in this study.

The exclusion criteria were as follows: (I) patients with pulmonary fissure dysplasia which could have led to segmentation failure; (II) patients with infectious lesion or atelectasis which prohibited identification of bronchi; (III) patients with a history of pulmonary surgery, including lobectomy and sublobectomy; (IV) the original scanned images greatly influenced by respiratory artifact which could have prohibited imaging analysis.

This study was approved by the Institutional Review Board of West China Hospital of Sichuan University,
Chengdu, China.

\section{Imaging analysis and $3 D$ reconstruction}

We downloaded the CT scan data from Picture Archiving and Communication Systems (PACS) in the form of Digital Imaging and Communications in Medicine (DICOM), and then imported the data into a $3 \mathrm{D}$ reconstruction system (IQQA-Lung, EDDA Technology, Princeton, NJ, USA) to generate patients' 3D segmental models. This system recognized the lung and automatically reconstructed a rough $3 \mathrm{D}$ model of the trachea-bronchi system. Minor misrecognitions and deficiencies in distal bronchi imaging were reconciled and replenished manually. Based on the $3 \mathrm{D}$ model of bronchial tree, the system could define the intersegmental planes interactively, and then build the segmental models. Meanwhile, since the intersegmental veins travel along the intersegmental planes, we also reconstructed the pulmonary veins in each $3 \mathrm{D}$ segmental model, and combined this with the pathways of intersegmental veins to ensure the accuracy of the segmental models (Figure S1).

\section{Definition of anatomic landmarks and lines on the lung surface}

The apex, diaphragmatic surface, costal surface, mediastinal surface, anterior margin, posterior margin, inferior margin, and pulmonary fissures are the known anatomic landmarks of the lung (9). In addition to these, we defined some other anatomic landmarks in this study: (I) the intersection point of the posterior margin and oblique fissure was defined as the posterior end of the oblique fissure (Figure 1A,B); (II) the intersection point of the anterior margin and the diaphragmatic surface of the left lung was defined as the left anteroinferior angle (Figure 1B); (III) the intersection point of the anterior margin of the right lung and horizontal fissure was defined as the right anteroinferior angle (Figure $1 B$ ); (IV) the intersection line of the oblique fissure and costal surface was defined as the costal margin (Figure 1B); (V) the intersection line of the horizontal fissure and costal surface was defined as the horizontal margin (Figure 1B); (VI) the intersection line of the oblique fissure and mediastinal surface was defined as the mediastinal margin (Figure 1C). Furthermore, we redefined the inferior margin and divided it into two parts: the anterior part, which is the inferior margin of the oblique fissure, and the posterior part, which is the remaining part of the inferior margin (Figure 1D). 

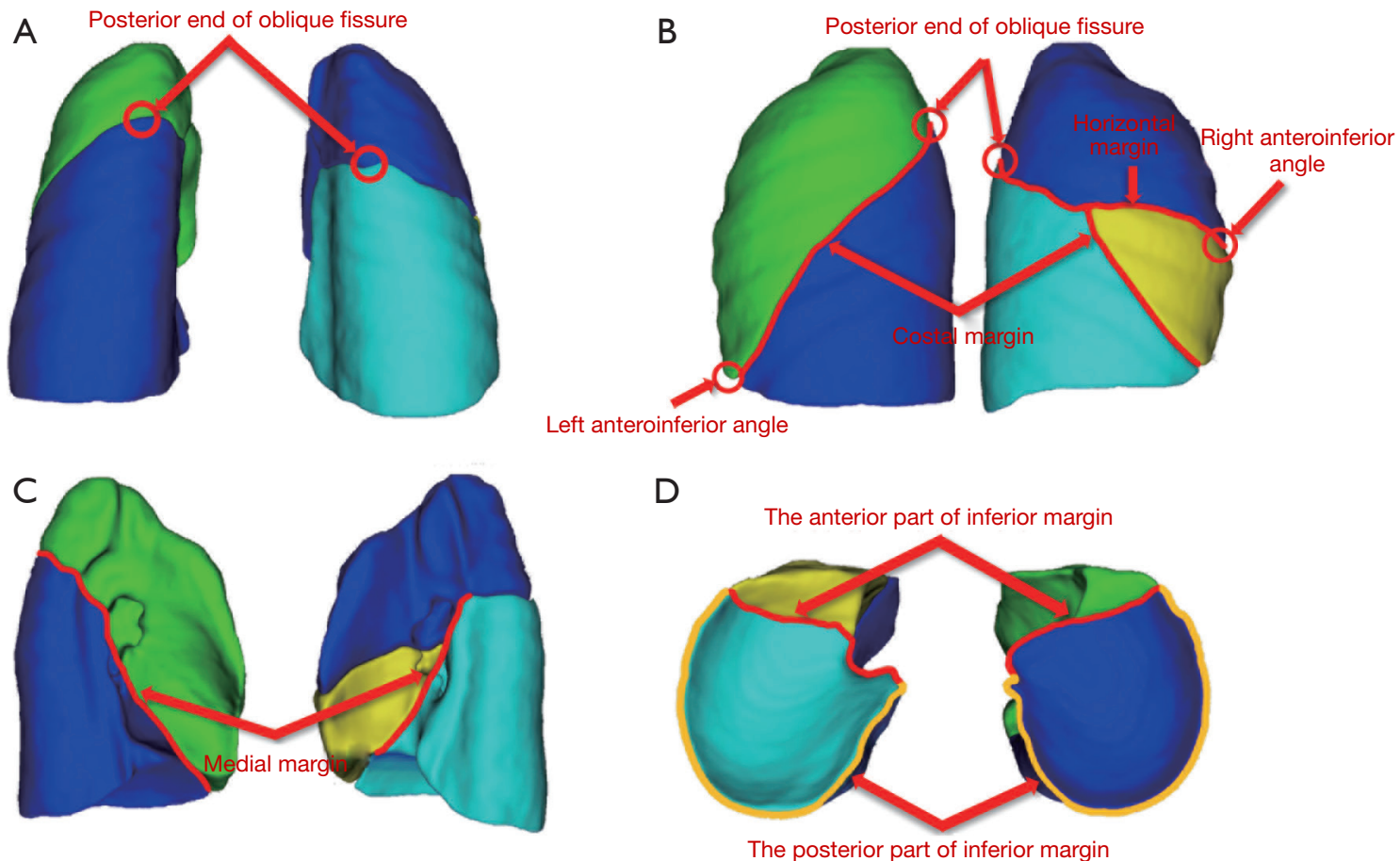

Figure 1 The anatomic landmarks of the lung surface. (A) The posterior view of the lung; (B) the lateral view of the lung; (C) the inferior view of the lung; (D) the medial view of the lung.

\section{Identification of the route of each segment on the lung surface along the lobe's anatomic landmark lines}

To identify the route of each segment on the lung surface, we firstly marked the intersegmental points between the adjacent segments along the lobe's anatomic boundary lines on 3D segment models.

The anatomic landmarks of the lobe and intersegmental points on the lung surface of the left upper lobe (LUL) are shown in Figure 2A. a is the apex of the left lung; b is the posterior end of the oblique fissure; $\mathrm{c}$ is the left anteroinferior angle; $\mathrm{n}$ is the intersection point of the costal margin and diaphragmatic surface; bn is the costal margin; ac is the anterior margin of the left lung; $d$ is the intersegmental point of the apicoposterior segment $(\mathrm{S} 1+2)$ and anterior segment (S3) on the anterior margin; e is the intersegmental point of $\mathrm{S} 1+2$ and superior lingual segment (S4) on the costal margin; $f$ is the intersegmental point of $\mathrm{S} 3$ and $\mathrm{S} 4 \mathrm{on}$ ac; and $\mathrm{g}$ is the intersegment point of $\mathrm{S} 1+2$, $\mathrm{S} 3$, and $\mathrm{S} 4$ on the costal surface.

The anatomic landmarks of lobe and intersegmental points on the lung surface of the left lower lobe (LLL) are shown in Figure 2B,C,D,E,F. b is the posterior end of oblique fissure; $\mathrm{m}$ is the intersection point of the mediastinal margin and diaphragmatic surface; $\mathrm{n}$ is the intersection point of the costal margin and diaphragmatic surface; bn is the costal margin; bw is the posterior margin of the LLL; $\mathrm{mv}$ is the mediastinal margin; $\mathrm{mn}$ is the anterior part of the inferior margin; mwn is the posterior part of the inferior margin; $h$ is the intersegmental point of the superior segment (S6) and anteromedial basal segment $(\mathrm{S} 7+8)$ on bn; i is the intersegmental point of $\mathrm{S} 6, \mathrm{~S} 7+8$, and the lateral basal segment (S9) on the costal surface; $j$ is the intersegmental point of S6, S9. And the posterior basal segment (S10) on the costal surface; $\mathrm{k}$ is the intersegmental point of $\mathrm{S} 6, \mathrm{~S} 7+8$, and $\mathrm{S} 10$ on the mediastinal surface; 1 is the intersegmental point of S6 and S10 on bw; o is the intersegmental point of $S 7+8$ and $S 9$ on mwn; $p$ is the intersegment point of $S 9$ and S10 on mwn; q is the intersegmental point of $S 7+8$ and $S 10$ on mwn; $r$ is the intersegmental point of $S 7+8, S 9$. and $S 10$ on the diaphragmatic surface; $s$ is the intersegmental point of $\mathrm{S} 6$ and $\mathrm{S} 7+8$ on mv.

The anatomic landmarks of the lobe and intersegmental points on the lung surface of the right upper lobe (RUL) are shown in Figure $3 \mathrm{~A}$. a is the apex of left lung; b is the posterior end of the oblique fissure; $\mathrm{c}$ is the right anteroinferior angle; $d$ is the intersection point of the 

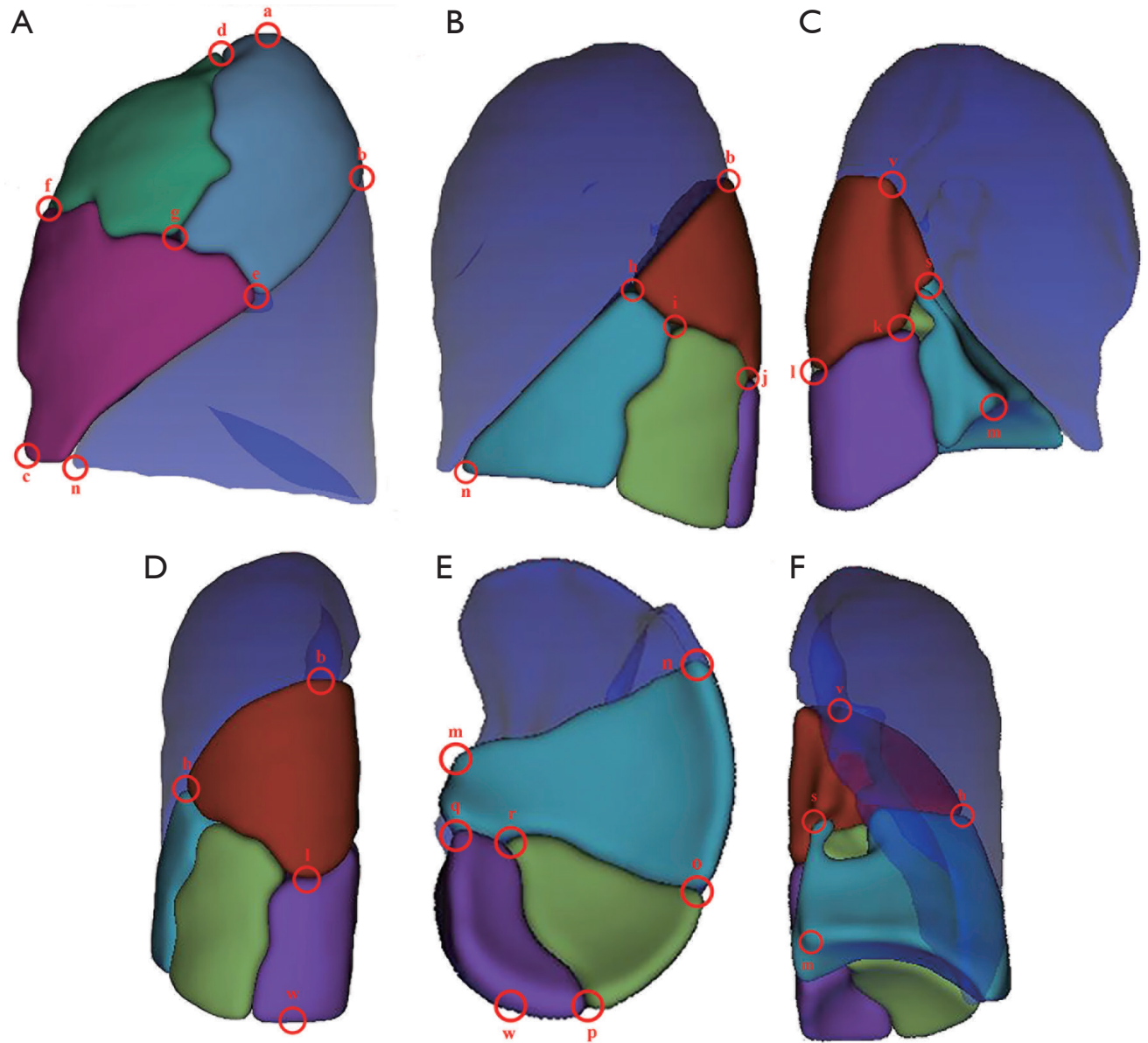

Figure 2 The anatomic landmarks and intersegmental marks of the left lung. (A) The lateral view of the LUL, which shows the anatomic landmarks and intersegmental marks on the costal surface of the LUL; (B) the lateral view of the LLL, which shows the anatomic landmarks and intersegmental marks on the costal surface of the LLL; (C) the medial view of the LLL, which shows the anatomic landmarks and intersegmental marks on the mediastinal surface of the LLL; (D) the posterior view of the LLL, which shows the anatomic landmarks and intersegmental marks on the posterior margin of the LLL; (E) the inferior view of the LLL, which shows the anatomic landmarks and intersegmental marks on the diaphragmatic surface of the LLL; (F) the anterior view of the LLL, which shows the anatomic landmarks and intersegmental marks on the oblique fissure. LUL, left upper lobe; LLL, left lower lobe.

costal margin and horizontal margin; ac is the anterior margin of RUL; $a b$ is the posterior margin of RUL; $c d$ is the horizontal margin; e is the intersegmental point of the apical segment (S1) and posterior segment (S2) on ab; $\mathrm{f}$ is the intersegmental point of $\mathrm{S} 1$ and $\mathrm{S} 3$ on ac; $g$ is the intersegmental point of $\mathrm{S} 2$ and $\mathrm{S} 3$ on cd.

The anatomic landmarks of the lobe and intersegmental points on the lung surface of the right lower lobe (RLL) are shown in Figure $3 B, C, D, E, F . \mathrm{b}$ is the posterior end of the oblique fissure; $\mathrm{m}$ is the intersection point of the mediastinal margin and diaphragmatic surface; $n$ is the intersection point of the costal margin and diaphragmatic surface; bn is the costal margin ; bw is the posterior margin of the LLL; $\mathrm{mv}$ is the mediastinal margin; $\mathrm{mn}$ is the anterior part of the inferior margin; mwn is the posterior part of the inferior margin; $h$ is the intersegmental point of S6 and the anterior basal segment (S8) on the bn ; i is the intersegmental point of S6, S8, and lateral S9 on the costal surface; $j$ is the intersegmental point of $S 6, S 9$, and $\mathrm{S} 10$ on the costal surface; $\mathrm{k}$ is the intersegmental point of S6, the medial basal segment (S7) and S10 on the mediastinal surface; 1 is the intersegmental point of $\mathrm{S} 6$ and $\mathrm{S} 10$ on bw; o is the intersegmental point of S8 and S9 on mwn; $p$ is the intersegment point of S9 and S10 

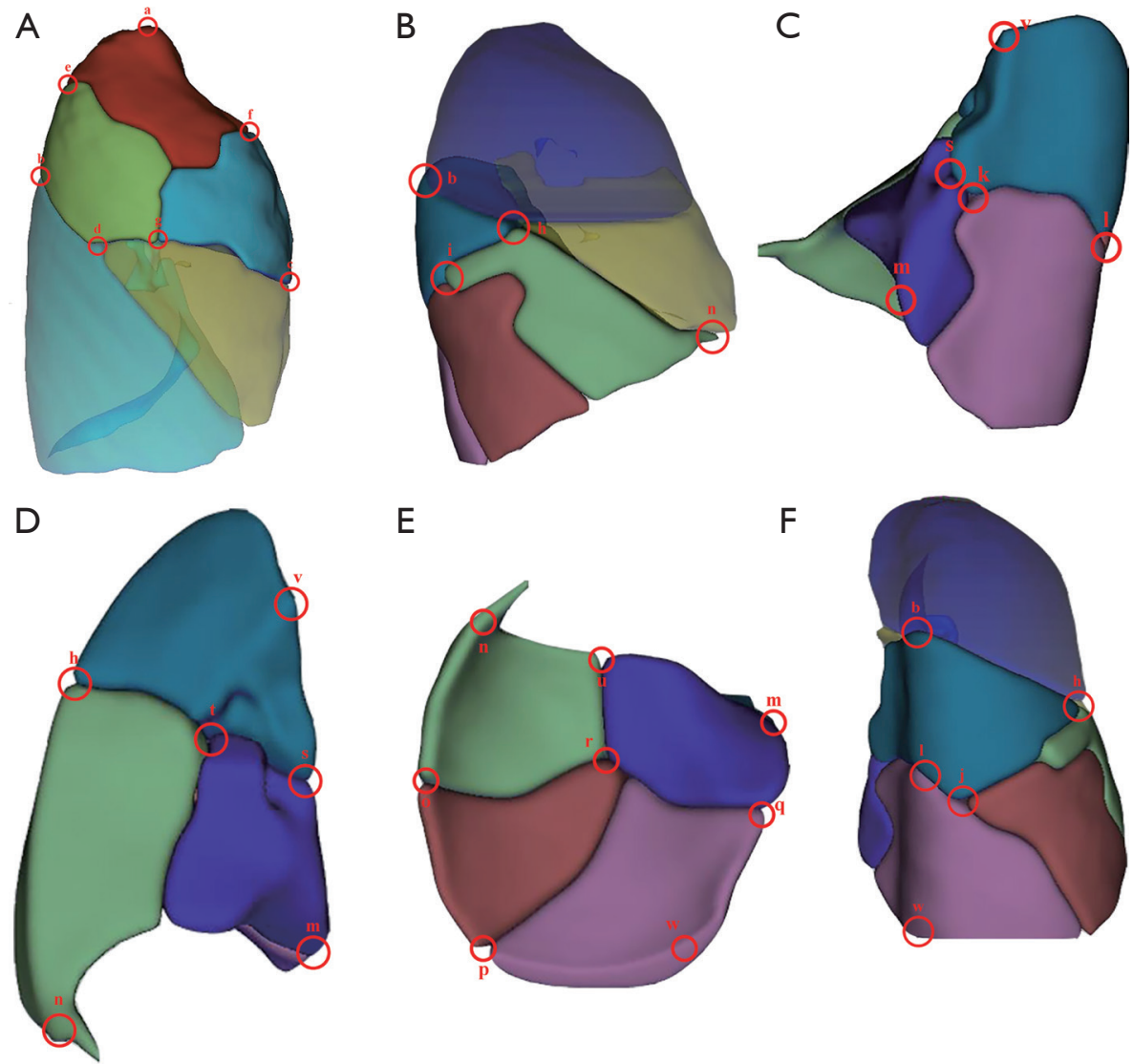

Figure 3 The anatomic landmarks and intersegmental marks of the RLL. (A) The lateral view of the RUL, which shows the anatomic landmarks and intersegmental marks on the costal surface of the RUL; (B) the lateral view of the RLL, which shows the anatomic landmarks and intersegmental marks on the costal surface of the RLL; (C) the medial view of the RLL, which shows the anatomic landmarks and intersegmental marks on the mediastinal surface of the RLL; (D) the posterior view of the RLL, which shows the anatomic landmarks and intersegmental marks on the oblique fissure; (E) the inferior view of the RLL, which shows the anatomic landmarks and intersegmental marks on the diaphragmatic surface of the RLL; (F) the anterior view of the RLL, which shows the anatomic landmarks and intersegmental marks on the posterior margin of the RLL. RLL, right lower lobe; RUL, right upper lobe.

on mwn; q is the intersegmental point of S7 and S10 on mwn; $\mathrm{u}$ is the intersegmental point of $\mathrm{S} 7$ and $\mathrm{S} 8$ on $\mathrm{mn} ; \mathrm{r}$ is the intersegmental point of S7, S8, S9, and S10 on the diaphragmatic surface; $s$ is the intersegmental point of S6 and $\mathrm{S} 7$ on mv; $\mathrm{t}$ is the intersegmental point of $\mathrm{S} 6, \mathrm{~S} 7$, and S8 on the oblique fissure surface.

The connecting line between two adjacent intersegmental points or between an intersegmental point and a lobe anatomic landmark was the route of each segment on the lung surface.

\section{Measurement of length of each segment on lung surface}

On the 3D segment models, we used the measurement tools in IQQA-Lung system to measure the length of each segment on the lung surface (Figures S2,S3). For instance, on the segment model of LUL, we measured the distance between a and $\mathrm{d}$ along the anterior margin of the left lung; thus the length of $\mathrm{S} 1+2$ on the anterior margin of left lung could be identified (Figure $S 2 A$ ).

\section{Statistical analysis}

All data were expressed as mean \pm standard deviations (SD). Intragroup differences of patients' baseline characteristics in the two groups were compared using $t$-test. A two-sided $\mathrm{P}$ value of $<0.05$ was considered statistically significant. All data analyses were conducted using SPSS software (version 
Table 1 Baseline characteristics of patients

\begin{tabular}{|c|c|}
\hline Characteristics & $\mathrm{n}$ \\
\hline \multicolumn{2}{|l|}{ Gender } \\
\hline Male & 241 \\
\hline Female & 259 \\
\hline \multicolumn{2}{|l|}{ Age, y } \\
\hline$<55$ & 270 \\
\hline$\geq 55$ & 230 \\
\hline \multicolumn{2}{|l|}{ Height, cm } \\
\hline$<160$ & 236 \\
\hline$\geq 160$ & 264 \\
\hline \multicolumn{2}{|l|}{ Weight, kg } \\
\hline$<60$ & 258 \\
\hline$\geq 60$ & 242 \\
\hline \multicolumn{2}{|l|}{ Smoke } \\
\hline Yes & 143 \\
\hline No & 357 \\
\hline \multicolumn{2}{|l|}{ Nodule location } \\
\hline LUL & 141 \\
\hline LLL & 68 \\
\hline RUL & 216 \\
\hline RML & 31 \\
\hline RLL & 97 \\
\hline \multicolumn{2}{|l|}{ Pleural traction } \\
\hline Yes & 74 \\
\hline No & 426 \\
\hline \multicolumn{2}{|l|}{ Complications } \\
\hline Yes & 234 \\
\hline No & 266 \\
\hline \multicolumn{2}{|l|}{ Complication type } \\
\hline COPD & 26 \\
\hline Hypertension & 74 \\
\hline Diabetes mellitus & 28 \\
\hline Tumor & 12 \\
\hline Other & 134 \\
\hline
\end{tabular}

n, number of patient. LUL, left upper lobe; LLL, left lower lobe; $\mathrm{RLL}$, right lower lobe; RUL, right upper lobe; RML, right middle lobe; COPD, chronic obstructive pulmonary disease.
22.0, SPSS Inc., Chicago, IL, USA).

\section{Results}

A total of 619 patients were enrolled in this study, 500 of whom (241 male and 259 female) had been successfully reconstructed with complete $3 \mathrm{D}$ segmental models. Seventy-two patients were excluded for pulmonary fissure dysplasia which could lead to segmentation failure or inaccuracy; 17 patients were excluded for infectious lesion or atelectasis which prohibits identification of bronchi; 22 patients were excluded for a history of pulmonary surgery; 8 patients were excluded for the low-quality original scanned images. The average age was $54.3 \pm 12.4$ years (range: 20-88 years), and there was no significant difference between males and females $(\mathrm{P}=0.624)$. The average height was $161.1 \pm 7.5 \mathrm{~cm}$ (range: $138-181 \mathrm{~cm}$ ). The average weight was $59.6 \pm 10.0 \mathrm{~kg}$ (range: $39-95 \mathrm{~kg}$ ). Baseline characteristics of patients are summarized in Table 1 .

The average length of each segment on lung surface is shown in Table 2. We did subgroup analysis according to gender, age, height, and weight. The results demonstrated that length of each segment on the lung surface had significant variability from patient to patient (Table 2).

Moreover, in order to reveal the spatial relationship among the segments and investigate the landmarks of intersegmental planes on the lung surface intuitively, we calculated the proportions of the lengths between adjacent segments along the lobe anatomic landmark lines (Table 3). The results of subgroup analysis stratified by gender, age, height, and weight are also shown in Table 3. Interestingly, there was no statistical difference in terms of proportions of length between adjacent segments even when stratified by gender, age, height, and weight. Thus, the position of intersegmental mark could be determined by the proportion of segmental length on the lung surface, which is detailed as follows.

\section{LUL (Figure 2A)}

The intersegmental point of $\mathrm{S} 1+2$ and $\mathrm{S} 3$ (d) was at about the superior $1 / 5$ of the anterior margin (ad); the intersegmental point of S3 and S4 (f) was at about the superior $3 / 5$ of the anterior margin (ad); the intersegmental point of $\mathrm{S} 1+2$ and $\mathrm{S} 4$ (e) was at about the superior $2 / 5$ of the costal margin (bn); the intersegmental point of $\mathrm{S} 1+2, \mathrm{~S} 3$, and $\mathrm{S} 4(\mathrm{~g})$ was at about the posterior 3/10 of ef. 
Table 2 The distance between the intersegmental marks on the lung surface

\begin{tabular}{|c|c|c|c|c|c|c|c|c|c|c|c|c|c|}
\hline \multirow[b]{2}{*}{ Variables } & \multirow[b]{2}{*}{$\begin{array}{c}\mathrm{N}, \text { mean } \\
\pm \mathrm{SD} \\
(\mathrm{mm})\end{array}$} & \multicolumn{12}{|c|}{ Subgroup analysis by gender, age, height, and weight } \\
\hline & & $\begin{array}{l}\text { Male, } \\
\text { mean } \\
\pm \mathrm{SD} \\
(\mathrm{mm})\end{array}$ & $\begin{array}{c}\text { Female, } \\
\text { mean } \\
\pm \mathrm{SD} \\
(\mathrm{mm})\end{array}$ & $\mathrm{P}$ & $\begin{array}{l}<55 \mathrm{y} \\
\text { mean } \\
\pm \mathrm{SD} \\
(\mathrm{mm})\end{array}$ & $\begin{array}{l}\geq 55 \mathrm{y} \\
\text { mean } \\
\pm \mathrm{SD} \\
(\mathrm{mm})\end{array}$ & $\mathrm{P}$ & $\begin{array}{l}<160 \\
\mathrm{~cm}, \\
\text { mean } \\
\pm \mathrm{SD} \\
(\mathrm{mm})\end{array}$ & $\begin{array}{l}\geq 160 \\
\mathrm{~cm}, \\
\text { mean } \\
\pm \mathrm{SD} \\
(\mathrm{mm})\end{array}$ & $\mathrm{P}$ & $\begin{array}{c}<60 \mathrm{~kg} \\
\text { mean } \\
\pm \mathrm{SD} \\
(\mathrm{mm})\end{array}$ & $\begin{array}{c}\geq 60 \mathrm{~kg} \\
\text { mean } \\
\pm \mathrm{SD} \\
(\mathrm{mm})\end{array}$ & $\mathrm{P}$ \\
\hline$A D$ & $56 \pm 19$ & $58 \pm 20$ & $55 \pm 18$ & 0.039 & $57 \pm 20$ & $56 \pm 18$ & 0.376 & $54 \pm 20$ & $58 \pm 18$ & 0.013 & $55 \pm 19$ & $59 \pm 19$ & 0.029 \\
\hline BE & $105 \pm 21$ & $108 \pm 21$ & $102 \pm 20$ & 0.004 & $106 \pm 22$ & $105 \pm 20$ & 0.633 & $104 \pm 21$ & $106 \pm 21$ & 0.242 & $104 \pm 21$ & $106 \pm 21$ & 0.401 \\
\hline EG & $60 \pm 37$ & $63 \pm 42$ & $58 \pm 3$ & 0.165 & $64 \pm 43$ & $57 \pm 32$ & 0.070 & $59 \pm 38$ & $61 \pm 36$ & 0.558 & $58 \pm 35$ & $62 \pm 40$ & 0.308 \\
\hline $\mathrm{HL}$ & $99 \pm 17$ & $101 \pm 18$ & $97 \pm 15$ & 0.008 & $100 \pm 19$ & $98 \pm 16$ & 0.347 & $98 \pm 16$ & $100 \pm 18$ & 0.260 & $98 \pm 16$ & $101 \pm 18$ & 0.039 \\
\hline $\mathrm{BH}$ & $100 \pm 18$ & $102 \pm 18$ & $98 \pm 17$ & 0.024 & $101 \pm 18$ & $99 \pm 17$ & 0.248 & $99 \pm 18$ & $101 \pm 17$ & 0.231 & $100 \pm 18$ & $100 \pm 18$ & 0.875 \\
\hline $\mathrm{HI}$ & $25 \pm 9$ & $26 \pm 9$ & $24 \pm 9$ & 0.045 & $26 \pm 9$ & $25 \pm 9$ & 0.269 & $24 \pm 9$ & $26 \pm 9$ & 0.186 & $24 \pm 9$ & $27 \pm 9$ & 0.005 \\
\hline $\mathrm{HJ}$ & $97 \pm 26$ & $98 \pm 26$ & $95 \pm 25$ & 0.148 & $98 \pm 25$ & $96 \pm 26$ & 0.500 & $94 \pm 23$ & $98 \pm 27$ & 0.165 & $96 \pm 26$ & $97 \pm 25$ & 0.845 \\
\hline MV & $190 \pm 23$ & $196 \pm 21$ & $185 \pm 24$ & $<0.001$ & $192 \pm 22$ & $189 \pm 24$ & 0.333 & $186 \pm 25$ & $193 \pm 21$ & 0.004 & $188 \pm 24$ & $193 \pm 22$ & 0.059 \\
\hline MS & $99 \pm 21$ & $102 \pm 20$ & $97 \pm 21$ & 0.031 & $101 \pm 22$ & $98 \pm 20$ & 0.211 & $98 \pm 22$ & $101 \pm 20$ & 0.197 & $99 \pm 21$ & $100 \pm 21$ & 0.504 \\
\hline SK & $43 \pm 15$ & $45 \pm 16$ & $42 \pm 14$ & 0.038 & $44 \pm 18$ & $43 \pm 13$ & 0.474 & $43 \pm 14$ & $43 \pm 16$ & 0.672 & $43 \pm 14$ & $44 \pm 16$ & 0.542 \\
\hline $\mathrm{OQ}$ & $119 \pm 15$ & $118 \pm 15$ & $119 \pm 15$ & 0.446 & $119 \pm 15$ & $118 \pm 15$ & 0.720 & $120 \pm 14$ & $118 \pm 15$ & 0.229 & $119 \pm 15$ & $118 \pm 14$ & 0.517 \\
\hline $\mathrm{BN}$ & $249 \pm 60$ & $259 \pm 82$ & $240 \pm 25$ & 0.001 & $250 \pm 28$ & $248 \pm 75$ & 0.750 & $248 \pm 86$ & $250 \pm 25$ & 0.729 & $249 \pm 79$ & $249 \pm 26$ & 0.968 \\
\hline$A C$ & $291 \pm 34$ & $304 \pm 34$ & $279 \pm 29$ & $<0.001$ & $293 \pm 36$ & $290 \pm 33$ & 0.481 & $283 \pm 34$ & $297 \pm 33$ & $<0.001$ & $289 \pm 34$ & $299 \pm 33$ & $<0.001$ \\
\hline BW & $178 \pm 26$ & $185 \pm 24$ & $171 \pm 26$ & $<0.001$ & $180 \pm 26$ & $176 \pm 26$ & 0.175 & $173 \pm 25$ & $181 \pm 26$ & 0.002 & $175 \pm 25$ & $181 \pm 26$ & 0.010 \\
\hline MWN & $288 \pm 37$ & $299 \pm 34$ & $276 \pm 37$ & $<0.001$ & $296 \pm 36$ & $282 \pm 37$ & $<0.001$ & $280 \pm 38$ & $294 \pm 36$ & $<0.001$ & $282 \pm 37$ & $294 \pm 37$ & 0.001 \\
\hline \multicolumn{14}{|c|}{ Right lung } \\
\hline $\mathrm{AE}$ & $53 \pm 15$ & $56 \pm 16$ & $50 \pm 14$ & $<0.001$ & $53 \pm 15$ & $54 \pm 16$ & 0.606 & $51 \pm 15$ & $55 \pm 15$ & 0.011 & $52 \pm 15$ & $54 \pm 15$ & 0.303 \\
\hline $\mathrm{CF}$ & $91 \pm 22$ & $93 \pm 24$ & $89 \pm 19$ & 0.033 & $90 \pm 21$ & $91 \pm 23$ & 0.648 & $92 \pm 21$ & $90 \pm 22$ & 0.455 & $90 \pm 21$ & $92 \pm 22$ & 0.430 \\
\hline$C G$ & $152 \pm 24$ & $158 \pm 24$ & $146 \pm 23$ & $<0.001$ & $152 \pm 23$ & $151 \pm 25$ & 0.943 & $146 \pm 23$ & $156 \pm 24$ & $<0.001$ & $148 \pm 25$ & $155 \pm 24$ & 0.004 \\
\hline $\mathrm{BL}$ & $71 \pm 14$ & $73 \pm 14$ & $70 \pm 14$ & 0.043 & $72 \pm 14$ & $71 \pm 14$ & 0.660 & $70 \pm 15$ & $73 \pm 13$ & 0.068 & $71 \pm 14$ & $72 \pm 14$ & 0.815 \\
\hline $\mathrm{HL}$ & $92 \pm 17$ & $94 \pm 20$ & $91 \pm 14$ & 0.237 & $93 \pm 19$ & $92 \pm 16$ & 0.793 & $92 \pm 17$ & $93 \pm 18$ & 0.733 & $92 \pm 17$ & $93 \pm 18$ & 0.554 \\
\hline
\end{tabular}

Table 2 (continued) 
Table 2 (continued)

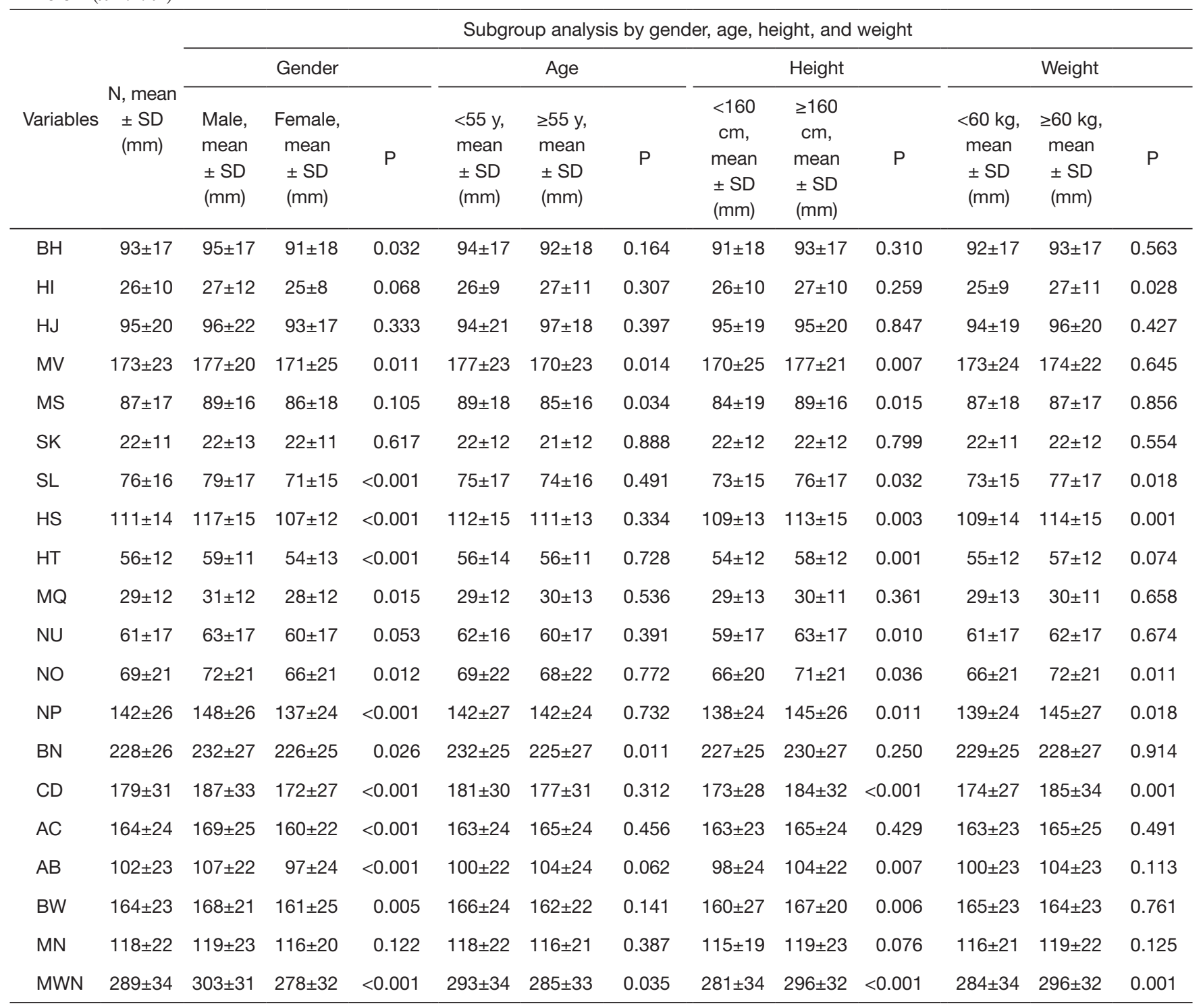

The variables in the first column represents distance between the intersegmental marks on the lung surface, and the details are shown in the section of Methods. N, all patients; SD, standard deviation.

\section{LLL (Figure 2B,C,D,E,F)}

The intersegmental point of S6 and $\mathrm{S} 7+8$ on the costal surface (h) was at about the superior $2 / 5$ of the costal margin (bn); the intersegmental point of $\mathrm{S} 6$ and $\mathrm{S} 7+8$ on the medial surface (s) was at about the midpoint of the mediastinal margin (mv); the intersegmental point of S6 and S10 (l) was at about the midpoint of the posterior margin of LLL (bw); the intersegmental point of S6, S7+8, and S9 (i) was at about the anterior $1 / 4$ of hl; the intersegmental point of S6, S9, and S10 (j) coincided with 1; the intersegmental point of $\mathrm{S} 6, \mathrm{~S} 7+8$, and $\mathrm{S} 10(\mathrm{k})$ was at about the anterior $1 / 5$ of sl; the intersegmental point of S7+8 and S10 (q) was at about the medial $1 / 10$ of the posterior part of the inferior margin (mwn); the intersegmental point of $\mathrm{S} 7+8$ and $\mathrm{S} 9$ (o) was at about the lateral $1 / 4$ of the posterior part of the inferior margin (mwn); the intersegmental point of S9 and S10 (p) was at about the midpoint of the posterior part of inferior margin (mwn); the intersegmental point of $\mathrm{S} 7+8$, $\mathrm{S} 9$, and S10 was at about the lateral 3/5 of oq. 
Table 3 The length proportion of the distance between the intersegmental marks to the anatomic landmarks

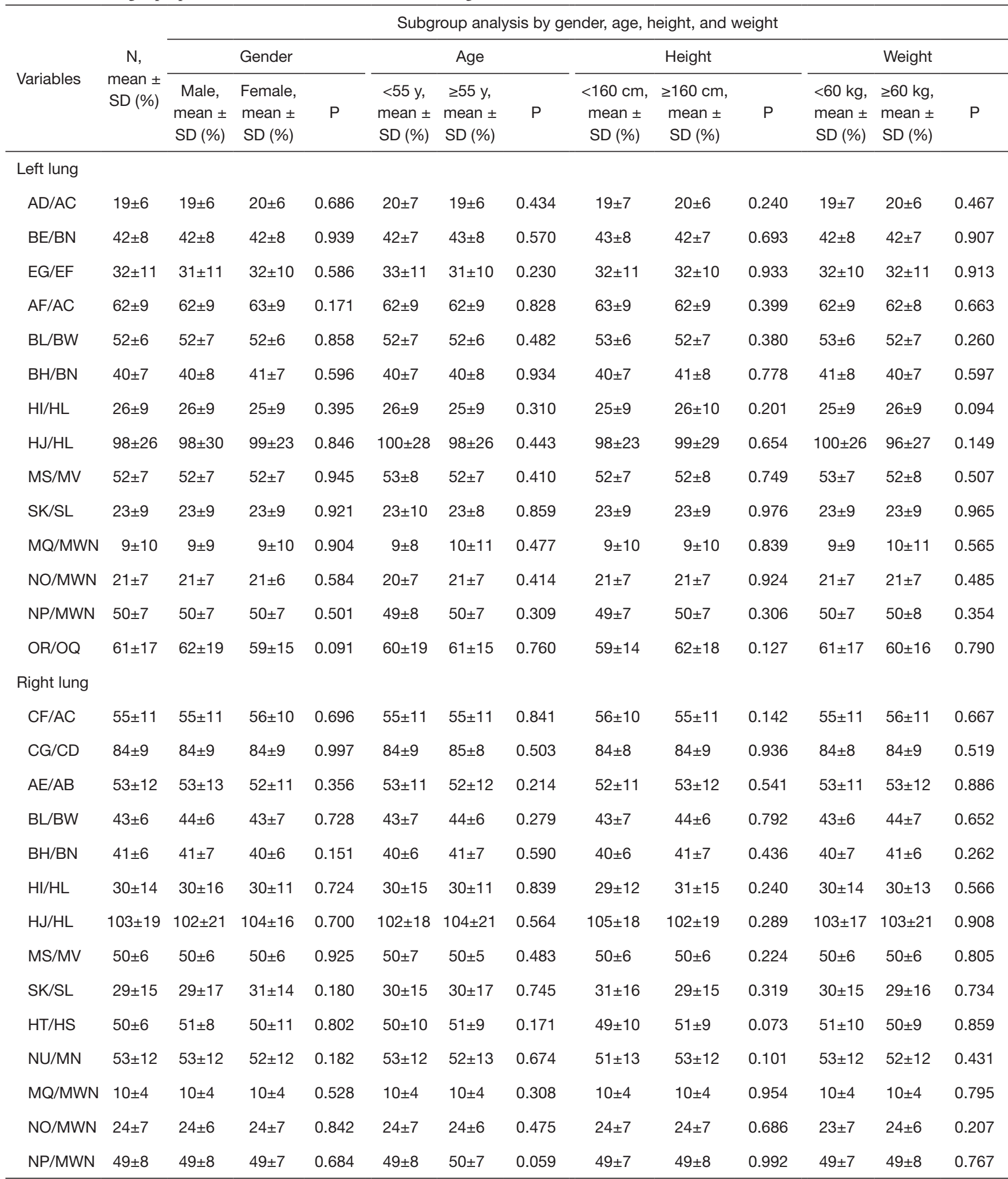

The variables in the first column represents distance between the intersegmental marks on the lung surface, and the details are shown in the section of Methods. N, all patients; SD, standard deviation. 


\section{RUL (Figure 3A)}

The intersegmental point of S1 and S2 (e) was at about the midpoint of the posterior margin of RUL (ab); the intersegmental point of S1and S3 (f) was at about the midpoint of the anterior margin of RUL (ac); the intersegmental point of S2 and S3 (g) was at about the posterior $1 / 5$ of the horizontal margin (cd).

\section{RLL (Figure 3B,C,D,E,F)}

The intersegmental point of S6 and S10 (1) was at about the superior $2 / 5$ of the posterior margin of the RLL (bw); the intersegmental point of S6 and S7 (s) was at about the midpoint of the mediastinal margin (mv); the intersegmental point of S6 and S8 (h) was at about the superior $2 / 5$ of the costal margin (bn) ; the intersegmental point of $\mathrm{S} 6, \mathrm{~S} 7$, and $\mathrm{S} 10(\mathrm{k})$ was at about the anterior $3 / 10$ of sl; the intersegmental point of S6, S8, and S9 (i) was at about the anterior $3 / 10$ of the hl; the intersegmental point of S6, S9, and S10 (j) coincided with 1; the intersegmental point of $S 6, S 7$, and $S 8$ (t) was at about the midpoint of sh; the intersegmental point of S7 and S8 (u) was at about the midpoint of the anterior part of the inferior margin $(\mathrm{mn})$; the intersegmental point of S7 and S10 (q) was at about the medial $1 / 10$ of the posterior part of inferior margin (mwn); the intersegmental point of S8 and S9 (o) was at about the lateral $1 / 4$ of the posterior part of the inferior margin (mwn); the intersegmental point of S9 and S10 (p) was at about midpoint of the posterior part of the inferior margin (mwn).

\section{Discussion}

Segmentectomy is anatomically more complicated than lobectomy, in which preoperative imaging assessment is the cornerstone. However, surgeons can only roughly generate an imaginary mode in mind to evaluate the resectability of the lesions with traditional two-dimensional (2D) CT images. 2D CT has been gradually replaced by $3 \mathrm{D}$ CT which provides stronger support for precise segmentectomy $(7,10,11)$. With the help of 3D CT, clinicians can localize the lung nodule, know the relevant bronchovascular relationships more accurately, and have prior knowledge of the anatomic variants, thus more efficiently estimating the surgical margin and determining the optimal procedure.

Furthermore, the most important issue that influences the implementation of segmentectomy is the identification of intersegmental planes. Unlike other organs that have their specific surface projections or morphological landmarks, such as the esophagus segmentation, there is no recognizable anatomic boundary between the adjacent pulmonary segments, which makes precise resection of the target segmental lung very difficult. A number of previous studies have reported different methods for identifying intersegmental planes (12-14). Surgeons usually adopt the inflation-deflation method to identify the surgical margin of the target segment (12). However, as a result of collateral ventilation, adjacent segments may also become inflated, obscuring the search for the intersegmental line, especially in patients with emphysema. Other studies have reported a method using infrared thoracoscopy after intravenous or intrabronchial injection of indocyanine green (ICG) to help with the identification of intersegmental lines $(13,14)$. However, tedious manipulations and the special equipment required hamper a broader use of this technique. These methods more or less have obvious limitations, and thus none have been regarded as the ideal method. Therefore, it is necessary to establish those lung intersegmental marks by which thoracic surgeons can easily and accurately identify the intersegmental planes.

We carried out this exploratory study to find suitable intersegmental marks on the lung surface. 3D segment models were reconstructed based on the bronchial tree in combination with the pathways of intersegmental veins. By measurement of the length of each segment on the lung surface along the lobe's anatomic lines, we acquired noteworthy findings, although the volume of each segment might have varied across individuals. On the basis of our results, compared to women, men have a relatively larger left lung (Table 2). In addition, the RLL became obviously atrophic over age (Table 2). Interestingly, despite of the differences in the absolute length among individuals, the proportions of length between adjacent segments on the lung surface along the lobe's anatomic lines stayed constant even through subgroup analysis. From this, we can conjecture that the constant length proportion revealed the lung intersegmental marks. This can possibly be considered as a surrogate mark to help surgeons identify the intersegmental line on the pleural surface effectively and easily, instead of using other complicated procedures or equipment. For example, when a thoracic surgeon plans to perform segmentectomy of left S1+2 (Figure S4, Table 3), the surgeon can determine the position of the intersegmental point between $\mathrm{S} 1+2$ and $\mathrm{S} 3$ (d) at the superior $1 / 5$ of the anterior margin (ac), the intersegmental point between $\mathrm{S} 1+2$, and $\mathrm{S} 4(\mathrm{e})$ at the superior $2 / 5$ of the costal margin 
(bn). Then, the surgeon should determine the position of the intersegmental point between S3 and S4 (f) at the superior $3 / 5$ of the anterior margin (ac), thus determining the position of the intersegmental point among $\mathrm{S} 1+2$ and $\mathrm{S} 3$ and $\mathrm{S} 4 \mathrm{~g}$ ) at the posterior $3 / 10$ of the line ef. At the moment, the intersegmental plane between $\mathrm{S} 1+2$ and $\mathrm{S} 3(\mathrm{dg})$ as well as the intersegmental plane between $\mathrm{S} 1+2$ and $\mathrm{S} 4(\mathrm{eg})$ can be easily determined.

This study has several limitations. First, for clinical convenience we approximated the constant proportions between these lung segments; for example, $49 \%$ was expressed as $1 / 2$. Second, the $3 \mathrm{D}$ segment models were reconstructed based on the $2 \mathrm{D}$ CT images acquired in the condition of lung inflation, while the lung was deflated during operation. However, since the length proportion stayed constant even when stratified by gender, age, height, and weight, it is conceivable that the length proportion was not heavily affected by the deflation of the lung. Third, the intersegmental planes identified by the marks have not been compared with the existing methods like intravenous injection of ICG, and these types of comprehensive studies should be conducted in the future.

The concept of "the lung surface intersegmental landmark" makes intangible intersegmental planes digitally identifiable. This novel concept might help solve the problem of determination of the boundary between pulmonary segments, which is considered as the most difficult aspect of anatomic segment dissection. It could guide surgeons to more easily find the intersegmental planes during anatomic segmentectomy, which would consequently reduce the dependence on special operative equipment, simplify operation manipulations, and shorten operation time with no additional cost. The discovery of lung surface intersegmental landmarks may also be valuable in the field of anatomy.

\section{Conclusions}

We discovered that proportion between lengths of adjacent segments on lung surface stayed constant. The constant proportion reflected and uncovered the lung surface intersegmental landmarks, which could navigate surgeons to identify intersegmental planes during anatomic segmentectomy in an easy and safe way without any cost.

\section{Acknowledgments}

Funding: This study was supported by the Chengdu Science and Technology Program Projects (2017-CY02-00029GX) (to Dr. L Liu), the 1.3.5 Project for Disciplines of Excellence, West China Hospital, Sichuan University (ZYJC18009) (to Dr. J Mei), and the Key Science and Technology Program of Sichuan Province, China (2016FZ0118) (to Dr. L Liu).

\section{Footnote}

Conflicts of Interest: The authors have no conflicts of interest to declare.

Ethical Statement: The authors are accountable for all aspects of the work in ensuring that questions related to the accuracy or integrity of any part of the work are appropriately investigated and resolved. This study was approved by the Institutional Review Board of West China Hospital of Sichuan University, Chengdu, China [No. 2019(982)].

\section{References}

1. Okada M, Koike T, Higashiyama M, et al. Radical sublobar resection for small-sized non-small cell lung cancer: a multicenter study. J Thorac Cardiovasc Surg 2006;132:769-75.

2. Kates M, Swanson S, Wisnivesky JP. Survival following lobectomy and limited resection for the treatment of stage I non-small cell lung cancer $<=1 \mathrm{~cm}$ in size: a review of SEER data. Chest 2011;139:491-6.

3. Kodama K, Higashiyama M, Okami J, et al. Oncologic Outcomes of Segmentectomy Versus Lobectomy for Clinical T1a N0 M0 Non-Small Cell Lung Cancer. Ann Thorac Surg 2016;101:504-11.

4. Dai C, Shen J, Ren Y, et al. Choice of Surgical Procedure for Patients With Non-Small-Cell Lung Cancer $\leq 1 \mathrm{~cm}$ or $>1$ to $2 \mathrm{~cm}$ Among Lobectomy, Segmentectomy, and Wedge Resection: A Population-Based Study. J Clin Oncol 2016;34:3175-82.

5. Cao C, Chandrakumar D, Gupta S, et al. Could less be more?-A systematic review and meta-analysis of sublobar resections versus lobectomy for non-small cell lung cancer according to patient selection. Lung Cancer 2015;89:121-32.

6. Kramer R, Glass A. XCVII. Bronchoscopic Localization of Lung Abscess. Annals of Otology, Rhinology \& Laryngology 1932;41:1210-20.

7. Iwano S, Yokoi K, Taniguchi T, et al. Planning of 
segmentectomy using three-dimensional computed tomography angiography with a virtual safety margin: Technique and initial experience. Lung Cancer 2013;81:410-5.

8. Chan EG, Landreneau JR, Schuchert MJ, et al. Preoperative (3-dimensional) computed tomography lung reconstruction before anatomic segmentectomy or lobectomy for stage I non-small cell lung cancer. J Thorac Cardiovasc Surg 2015;150:523-8.

9. Drake RL, Vogl W, Mitchell AW. Gray's anatomy for students (3rd ed.). Edinburgh: Churchill Livingstone/ Elsevier, 2014:167-74.

10. Fukuhara K, Akashi A, Nakane S, et al. Preoperative assessment of the pulmonary artery by three-dimensional computed tomography before video-assisted thoracic surgery lobectomy. Eur J Cardiothorac Surg 2008;34:875-7.
11. Oizumi H, Kanauchi N, Kato H, et al. Anatomic thoracoscopic pulmonary segmentectomy under 3-dimensional multidetector computed tomography simulation: a report of 52 consecutive cases. J Thorac Cardiovasc Surg 2011;141:678-82.

12. Okada M, Mimura T, Ikegaki J, et al. A novel video-assisted anatomic segmentectomy technique: selective segmental inflation via bronchofiberoptic jet followed by cautery cutting. J Thorac Cardiovasc Surg 2007;133:753-8.

13. Misaki N, Chang SS, Igai H, et al. New clinically applicable method for visualizing adjacent lung segments using an infrared thoracoscopy system. J Thorac Cardiovasc Surg 2010;140:752-6.

14. Oh S, Suzuki K, Miyasaka Y, et al. New technique for lung segmentectomy using indocyanine green injection. Ann Thorac Surg 2013;95:2188-90.
Cite this article as: $\mathrm{Xu} \mathrm{Y,} \mathrm{Gan} \mathrm{F,} \mathrm{Xia} \mathrm{C,} \mathrm{Wang} \mathrm{Z,} \mathrm{Zhao} \mathrm{K,} \mathrm{Li} \mathrm{C,}$ Mei J, Liu C, Liao H, Pu Q, Liu L. Discovery of lung surface intersegmental landmarks by three-dimensional reconstruction and morphological measurement. Transl Lung Cancer Res 2019;8(6):1061-1072. doi: 10.21037/tlcr.2019.12.21 


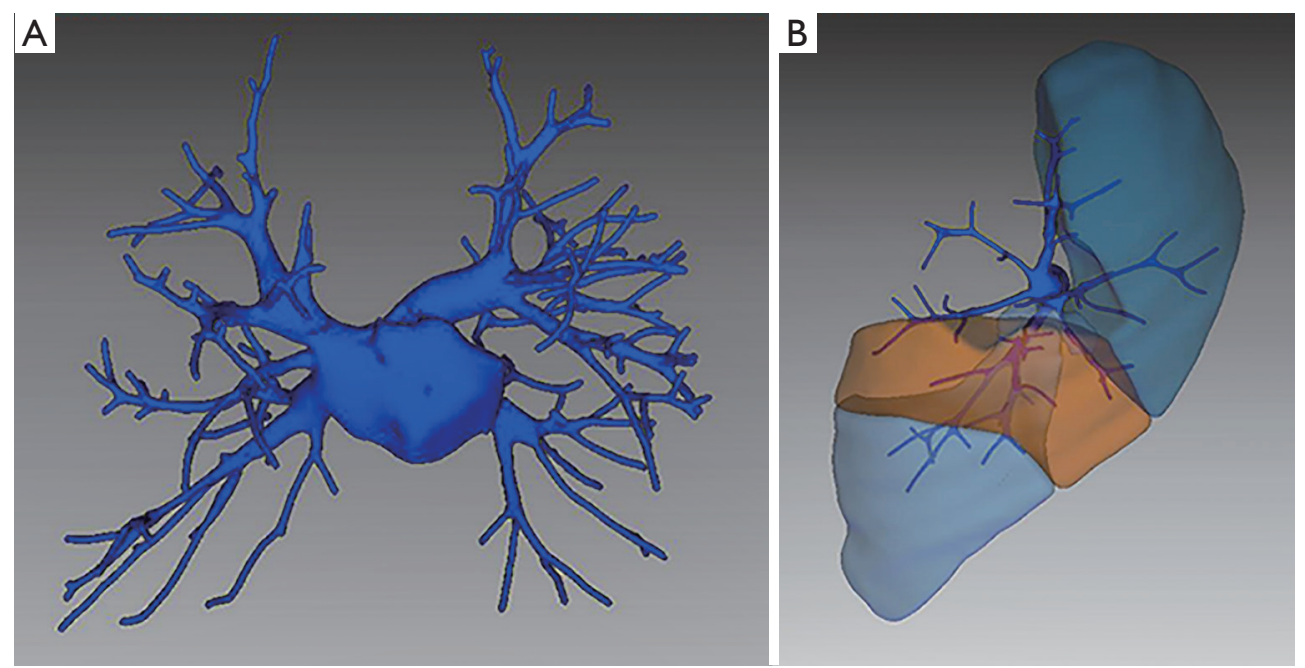

Figure S1 3D model of pulmonary vein. (A) 3D morphology of the pulmonary vein; (B) lateral view of the LUL after hiding S3. LUL, left upper lobe.
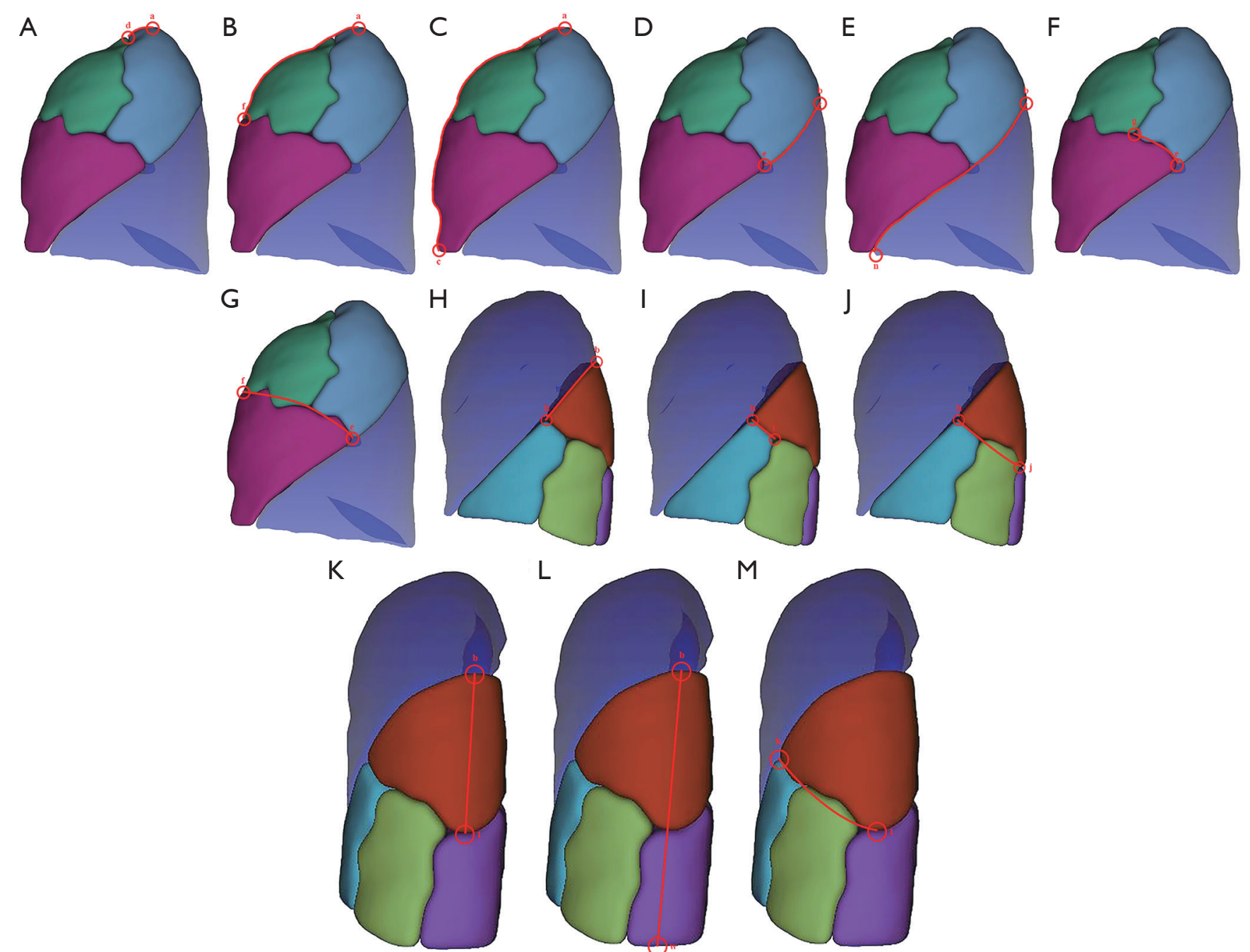

$N$

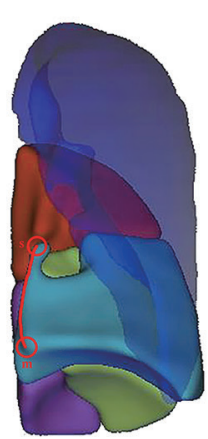

R

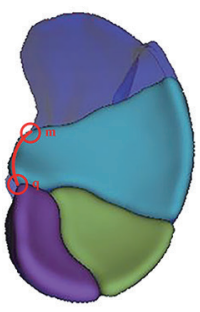

S

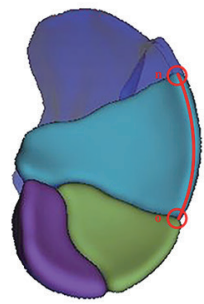

○

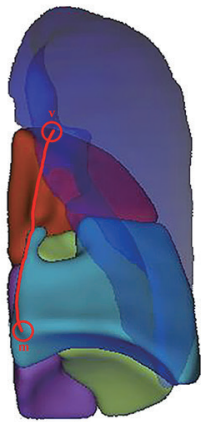

$\mathrm{T}$

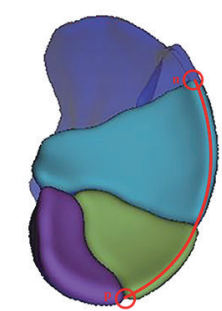

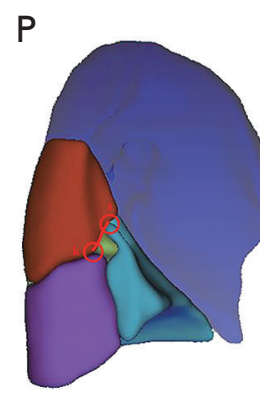

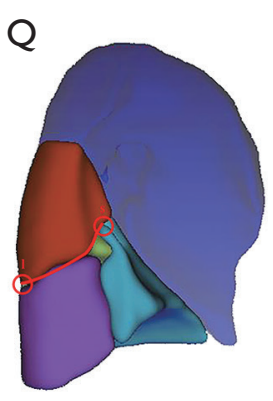

U

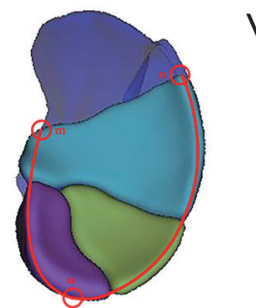

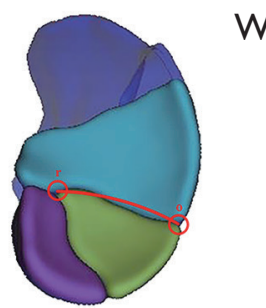

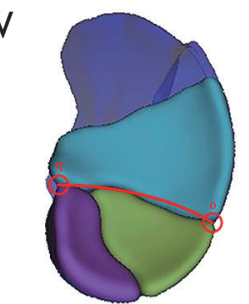

Figure S2 Diagrammatic measurements of each distance between the intersegmental marks on the surface of the left lung on 3D lung segment models. 
